\title{
A Review of Video Modelling and Scripts in Teaching Conversational Skills to Individuals with Autism Spectrum Disorders
}

\author{
Cheong Ying Sng • Mark Carter • Jennifer Stephenson
}

Received: 6 April 2013 / Accepted: 29 August 2013 / Published online: 4 December 2013

(C) Springer Science+Business Media New York 2013

\begin{abstract}
Individuals with autism spectrum disorders (ASD) have difficulties acquiring social skills without direct intervention. This article provides a review of research on the use of video modelling and scripts to improve the verbal conversational skills of individuals with ASD. The studies reviewed included participants with a diagnosis of ASD and reported empirical data on some aspect of verbal conversation. Percentage of nonoverlapping data and percentage exceeding the mean metrics were calculated for each study and compared. Maintenance and generalisation data were also extracted. The results suggest that scripted interventions may be as effective as video modelling interventions and audio scripts may be more effective than visual scripts. Most studies focussed on relatively simple aspects of conversation such as initiating or responding. Nevertheless, the body of research is limited and further studies are needed to clarify these findings. Recommendations for future research and the limitations of this review are discussed.
\end{abstract}

Keywords Video modelling · Scripts · Conversation ·

Review $\cdot$ Autism spectrum disorder

Autism and related pervasive developmental disorders are developmental impairments characterised by difficulties in social interaction, communication, and behavioral inflexibility (American Psychiatic Association 2000). Given that social interaction, social cognition, and related social use of communication are areas of deficit in autism spectrum disorders (ASD), acquiring appropriate conversation skills may present significant difficulties for these individuals (Fine et al. 1994).

Many children with high-functioning ASD do develop sophisticated language abilities (Rubin and Lennon 2004).

C. Y. Sng $(\bowtie) \cdot$ M. Carter $\cdot$ J. Stephenson

Macquarie University Special Education Centre, Macquarie

University, Building X5A, North Ryde, NSW 2109, Australia

e-mail: cheong.sng@mq.edu.au
Despite this ability, children with ASD tend to use developmentally basic conversation strategies such as repetitions to engage in dialogue, but are more likely to make irrelevant comments and fail to respond to queries (Capps et al. 1998). Individuals with ASD may often appear to be uninterested in social interaction and may find it difficult to comprehend the subtleties in social messages (Weiss et al. 2009). Although some children with ASD may have seemingly extensive vocabularies, they may struggle to cope with deciphering the complex social messages that characterise verbal conversation (Twachtman-Cullen 2000). Conversation requires constant monitoring of the changing social environment and the ability to adapt one's behavior to cater for this change within the constraints of acceptable social conventions (Carpenter and Tomasello 2000; Klinger and Williams 2009). This constant exchange of information may be confusing to individuals with ASD and they may be challenged by the nuances of social conversation (Carter et al. 2005; Weiss et al. 2009). Rutter (1978) observed that children with autism often give the impression that they are talking to rather than talking with others. This is reflected in an absence of the reciprocal interaction between conversational partners that characterises typical conversations (Rutter 1978).

Conversational skill involves a web of actions and reactions that requires the individual to have an understanding of the partner's interests and prior knowledge (Baron-Cohen 1995; Carpenter and Tomasello 2000; Dixon et al. 2009; Machalicek et al. 2008). An individual should be aware of the information the listener needs on a topic, what to say, how to say it clearly, how much to say, and the way to convey the information. Individuals with ASD have difficulties in all of these areas (Twachtman-Cullen 2000). Limitations in social cognition or perspective taking may also hinder the conversational ability of a person with ASD (Dixon et al. 2009). Such skills are important because interpersonal interactions often hinge on the state of mind of the person with whom you are 
interacting, and understanding the state of mind of other persons presents specific difficulties for individuals with ASD (Baron-Cohen 1995). The inability to read social cues and effectively "predict" what the other person is interested in can seriously impede social interactions (Klinger and Williams 2009). There is evidence that, despite their social impairment, individuals with autism long for interaction with their peers but may have limited ability to initiate and reciprocate (Church et al. 2000; Shea and Mesibov 2005).

The various capacities and skills that enable conversation are typically learned incidentally, often without any conscious effort, through joint attention and social interaction (Carpenter and Tomasello 2000; Klinger and Williams 2009). This is often not the case for individuals with ASD. Designers of interventions that target conversational skills often attempt to break down this complex activity into smaller components or discrete trials in order to allow the learner to build on individual constituents with each additional skill learnt (Charlop et al. 2009). Although numerous studies have been conducted using a variety of methods to improve the social skills of children with ASD and many reviews have been published addressing the efficacy of social skills interventions (e.g., Matson et al. 2007; Scattone 2007; Stichter et al. 2007; Williams et al. 2007), they have not specifically focused on analysing interventions addressing conversational skills as a subset of social skills.

Two promising specific intervention approaches that have been developed over the past two decades to address the social communication needs of children with ASD are script training and video modelling. Scripts are a prompting strategy often used to teach social skills to individuals with ASD. They can be visual or auditory and are usually faded out to avoid overreliance. Scripts can act as a prompt for an interaction or can give an example of an appropriate verbal exchange in a predetermined situation. The use of scripts allows routines to be taught step-by-step in adherence to social rules, which may not be obvious to individuals with ASD (Klinger and Williams 2009). Charlop-Christy and Kelso (2003) suggested that the use of written prompts could encourage individuals with reading skills to engage in conversation with others. Thus, scripts seem to have considerable potential in facilitating conversational exchange.

A video-modelled intervention demonstrates a desired behaviour and the student imitates the behavior shown. The video may be of the student performing the behavior (selfmodelling) or of another person. Video modelling explicitly shows how an individual behaves in a given situation and is a strategy that may allow individuals who lack reading skills an opportunity to acquire the skills needed to engage in social interactions. A video-modelled intervention has several features that may make it suitable for the teaching of conversational skills. The interventionist may use video recordings to replay scenarios, highlight the discriminative stimuli, and include demonstration of features of conversation such as appropriate volume, speech prosody, and inflection (Charlop et al. 2010; Charlop-Christy et al. 2000; Ferraioli and Harris 2011). In particular, video modelling provides a concrete demonstration of what is required and how the behavior should be performed. Thus, both scripts and video modelling appear to have features that would make them suitable for teaching conversational skills to individuals with ASD. The present review examines both the efficacy of these interventions and the limitations of the existing research. Possibilities for future research are then discussed.

\section{Method}

\section{Identification of Studies}

Searches were conducted in June 2013 in ERIC, PsycINFO and CINAHL using a combination of several search terms. Initially, a broad search was conducted using the terms "autis" or "ASD" combined with one of the following terms individually; "conversation", "social skills", "communication", "social interaction", "topic shift", "topic maintenance", or "pivotal response". No restriction was placed on the publication date, language, or type of article. Duplicated articles were identified and excluded. Ancestral searches of the shortlisted articles were conducted to locate additional articles. This process identified 358 articles. Studies that employed a video-modelling or script-training intervention were then identified.

The abstract and title of each article were examined. Studies were included in this review if they (a) included participants with autism spectrum disorder; (b) employed empirical methods; (c) reported quantitative data relating to a specific measure of conversation; (d) used video modelling, script training, or a combination as an intervention; and (e) were available in English. Once studies were shortlisted based on the above criteria, the entire study was examined. Studies with multiple dependent variables were included only if data could be extracted for conversational outcomes. Conversation was defined as a verbal exchange of social information. Studies where verbal initiations were measured were excluded from this review if the initiation was instrumental (i.e., to accept, request, or reject an object or service) or was unable to be separated from gestural initiations. Studies where verbal utterances were measured when the participant was engaged in pretend play with inanimate objects (D'Ateno et al. 2003) or playing a role other than themselves (MacDonald et al. 2005) were excluded, as exchanges with objects in pretend play or the assumption of a different persona are not typical of conversational exchanges.

Interrater Reliability on Article Inclusion

A list of all the studies and abstracts identified in the searches was compiled. The first author examined each abstract to 
determine if the study satisfied the specified criteria. The second and third authors each independently examined half of the abstracts, respectively, against the criteria for inclusion. Reliability was calculated by dividing the number of agreements by the total number of articles and multiplying by 100 . Reliability was $92.8 \%$. All disagreements were discussed and resolved by examining the full article. Twenty-five articles were identified as suitable for inclusion.

\section{Data Extraction}

The following information for each study was extracted by the first author: intervention type (script or video modelling); type of script (audio or visual); type of model (adult/peer/self/ "point of view"/other); research design; participant age (years); number of male and female participants; diagnosis; diagnostic instrument used; agency or individual completing the diagnosis; and intervention setting (mainstream school/ special school/after school program/home/clinic). Data were also extracted on the presence of generalisation data (across stimuli, settings, or people); maintenance data; social validity evaluation; treatment integrity; interrater reliability; additional reinforcers; and treatment fading. A random number generator was used to identify six (24\%) articles, which were used for the purposes of interrater reliability. The first and second authors extracted the relevant data independently and any disagreements were discussed. Reliability was calculated by dividing the number of agreements by the number of agreements and disagreements and multiplying by 100 . Interrater reliability for data extraction was $95.7 \%$. Summary descriptive information was extracted by the first author on the participants, dependent variables, procedures for evaluating social validity, and interrater reliability findings for the six randomly selected studies. The second author examined the description and original article for the six randomly selected studies to determine whether the summary was accurate. The summary data were assessed as accurate in $94.9 \%$ of instances. Again, discrepancies were resolved by consensus.

In addition, as a measure of research quality, the first and second authors examined all 25 studies to determine whether or not they provided at least three clear demonstrations of experimental control at different points in time, as recommended by Horner et al. (2005). The reliability for estimates of experimental control was $84 \%$. Disagreements were resolved by discussion with all three authors.

\section{Study Metrics}

Traditionally, conclusions on the efficacy of single-subject studies have been interpreted in terms of both experimental control and magnitude of effect by subjective visual inspection of the graphed data (Carter 2013). Scruggs et al. (1987) proposed using the percentage of non-overlapping data (PND) statistic to allow for a more objective evaluation of singlesubject studies. The PND is simply a mathematical computation of the number of data points above the highest data point (or lowest data point in the case of decreasing behaviors) in the baseline phase divided by the total number of data points in the same phase (Scruggs et al. 1987). One critical limitation of PND is if baseline data reaches ceiling level for interventions seeking to increase behavior or floor level, in the case of decreasing behavior, the PND is automatically zero. This calculation may be inconsistent with interpretations drawn from visual inspection of the data regarding the magnitude of treatment effects. To avoid this problem a percentage of data points exceeding the median of the baseline phase (PEM) has been proposed (Ma 2006). The PEM is a calculation of the number of data points in a treatment phase that are above (or below if the behavior decreases) the median data in the baseline phase (Ma 2006). PEM differs from PND solely on the chosen referent data point in the baseline phase from which one would base an assessment of whether there has been a response to the intervention. A wide range of metrics have been proposed for meta-analytic synthesis of single-case research and there is no current consensus on the most appropriate measures (Gage and Lewis 2012). While PND has a number of acknowledged technical limitations, it remains the most widely used metric for evaluating single-subject research, providing a basis for interpretation of effects. Noting these outstanding issues, it can be argued that if multiple approaches (such as PND and PEM) tend to converge on similar conclusions, stronger inferences may be drawn regarding the efficacy of interventions (Preston and Carter 2009).

In this review, both PND and PEM statistics were calculated for each of the studies comparing the baseline phase to an intervention phase. Where a return to baseline design was implemented to demonstrate experimental control (e.g., Lee 2007; Tetreault and Lerman 2010), adjacent phases were individually compared in accordance with the recommendation of Parker and Hagan-Burke (2007). Thus, where an $\mathrm{ABAB}$ design was used, metrics were calculated comparing $\mathrm{A} 1$ to $\mathrm{B} 1, \mathrm{~B} 1$ to $\mathrm{A} 2$, and finally, $\mathrm{A} 2$ to $\mathrm{B} 2$.

In a number of studies (Brown et al. 2008; Charlop et al. 2009; Charlop and Milstein 1989; Charlop-Christy and Kelso 2003; Charlop-Christy et al. 2000; Davis et al. 2010; Haymes 1995; Lee 2007; Pollard et al. 2012; Sansosti and PowellSmith 2008; Tetreault and Lerman 2010) generalisation probes were performed during the baseline phase. In these cases, generalisation data collected during the intervention were compared with baseline data. In the majority of cases where generalisation data were available, no baseline generalisation probe data were available. In these instances, metrics were calculated for treatment data only and then again for treatment and generalisation data. Comparing these figures allows for judgement whether generalisation data reduced or increased the relevant treatment metric. A similar comparison 
was conducted for maintenance data by comparing treatment data alone to treatment plus maintenance data.

Where possible, in cases where an additional tangible reward was offered as reinforcement, PND and PEM statistics were calculated for the original treatment and also for the combined treatment. Sherer et al. (2001) made comparisons of using "self" or "other" as a model in their treatment design. In this instance, three separate calculations were done: "self" as a model, "other" as a model, and a study-wide statistic. When analysing data, the metrics for "self" and "other" as a model were separated and compared with the studies implementing the same form of intervention. In studies where there were two or more participants or dependent variables, an individual participant metric was calculated for each dependent variable. A study-wide mean was then calculated for the same dependent variables to ensure equivalent comparisons. The same method was applied for the calculation of PND and PEM.

A random number generator was used to identify eight studies (32\%) for determining interrater reliability for PND and PEM calculations. The first and the second authors calculated the PEM and PND statistics for these studies independently. The percentage of agreements was calculated by dividing the number of agreements by the total number of statistics and multiplying by 100. A total of 212 calculations were completed, and interrater reliability for the PND statistic was $95.3 \%$ and for PEM it was $94.4 \%$. The disagreements typically related to very small or complex graphs and were due either to miscounting the number of data points or to a disagreement on whether a data point lay below or above the baseline benchmark. A Pearson correlation statistic was calculated between the first and second authors for both PND and PEM and found to be 0.999 and 0.994 , respectively, suggesting that disagreements had a very minor impact on the value of calculated metrics.

\section{Results}

\section{Studies}

The studies were categorised as addressing video modelling or scripts. A video-modelled intervention was used in 13 studies including 2 using a video-modelled Social Story ${ }^{\circledR}$ (Sansosti and Powell-Smith 2008; Scattone 2008). The studies that employed video modelling were analysed according to whether they included self as a model $(n=1)$, a peer of a similar age model $(n=4)$, an adult model $(n=7)$, other as a model $(n=1)$ or "point of view" where the video was shown from the participant's perspective $(n=1)$ was used. A comparison of the effect of using a peer as a model against the participant as a model was conducted in one study and excerpts from a Disney cartoon were used as the other model in one study. Scripts were employed as an intervention in 10 studies. Thiemann and
Goldstein (2001) used a combination of Social Stories ${ }^{\circledR}$, text, picture cues, and video feedback in their study. A summary of the number of participants, research design, presence of generalisation or maintenance data, and the intervention used in the video-modelled studies is presented in Tables 1 and 2 . Parallel information for studies employing scripts is presented in Tables 3 and 4.

\section{Participants}

In total, there were 69 participants, including 60 males and 9 females. Their ages ranged from 4 to 20 years and 7 months at the commencement of the study. Twenty-nine participants (27 males, 2 females) ranging in age from 4 to 11 years and 3 months were involved in video-modelled interventions. There were 40 participants in 12 studies where scripts were used as an intervention. Thirty-three of those participants were male and seven were female, ranging in age from 4 to 20 years and 7 months.

All but two participants presented with a diagnosis of ASD. There were five participants in the study conducted by Thiemann and Goldstein (2001), two of whom were considered socially impaired but did not have a formal diagnosis of ASD. One of the two participants without a formal diagnosis of autism scored in the mild to moderate range on the Childhood Autism Rating Scale (CARS) while the other one did not. The data for the participant without autism was excluded from the results in this review.

Information regarding the instrument used for diagnosis was reported in $60 \%$ of the studies. Information on the degree of autistic symptomatology was provided using CARS for 14 participants (20.3\%) in four studies (Davis et al. 2010; Sherer et al. 2001; Tetreault and Lerman 2010; Thiemann and Goldstein 2001). The degree of autism was not reported in 14 studies (38 participants, $55.1 \%$ ) but information on IQ, adaptive behavior or language on formal test data performed on normed instruments was provided. Anecdotal data was reported in five studies for 13 participants (Charlop et al. 2009; Lee 2007; Pollard et al. 2012; Taylor et al. 1999; Wichnick et al. 2010), and for two studies, there was no reported information on the participants' abilities (Buggey 2005; Ogletree et al. 1995).

\section{Interobserver Reliability}

Interobserver reliability was reported for all except one of the studies (Ogletree et al. 1995). Mean interobserver reliability ranged from 82 to $100 \%$, exceeding the conventionally accepted minimum of $80 \%$ (Horner et al. 2005). One researcher did not report the number of sessions used in the reliability rating. All other studies reported interobserver reliability data for $20 \%$ or more of sessions undertaken, conforming to the conventional minimum standard and 
Table 1 Summary of design and participants in studies using video modelling

\begin{tabular}{|c|c|c|c|c|c|c|c|c|}
\hline \multirow[t]{2}{*}{ Authors } & \multirow[t]{2}{*}{ Intervention } & \multirow[t]{2}{*}{ Design } & \multicolumn{2}{|c|}{ Participant } & \multirow[t]{2}{*}{$\begin{array}{l}\text { Age } \\
\text { (years) }\end{array}$} & \multirow[t]{2}{*}{ Participant details } & \multicolumn{2}{|c|}{$\begin{array}{l}\text { Diagnostic } \\
\text { information }\end{array}$} \\
\hline & & & Male & Female & & & Tool & Agent \\
\hline Apple et al. (2005) & $\begin{array}{l}\text { Video modelling- } \\
\text { peer as model }\end{array}$ & $\begin{array}{l}\text { Multiple baseline across } \\
\text { participants }\end{array}$ & 2 & & 5 & $\begin{array}{l}\text { PPVT III score of } \\
\quad 86-119\end{array}$ & $\checkmark$ & $\checkmark$ \\
\hline Buggey (2005) & $\begin{array}{l}\text { Video modelling- } \\
\text { self as model }\end{array}$ & $\begin{array}{l}\text { Multiple baseline across } \\
\text { participants }\end{array}$ & 2 & & $9-13$ & None reported & $x$ & $\checkmark$ \\
\hline $\begin{array}{l}\text { Charlop and Milstein } \\
\text { (1989) }\end{array}$ & $\begin{array}{l}\text { Video modelling- } \\
\text { adult as model }\end{array}$ & $\begin{array}{l}\text { Multiple baseline across } \\
\text { participants and } \\
\text { multiple probe across } \\
\text { conversations }\end{array}$ & 3 & & $6-7$ & $\begin{array}{l}\text { PPVT, SIT, Leiter: } \\
\text { mental age range } \\
3 \text { years and } \\
10 \text { months to } \\
6 \text { years } 6 \text { months }\end{array}$ & $\checkmark$ & $x$ \\
\hline Charlop et al. (2009) & $\begin{array}{l}\text { Video modelling- } \\
\text { adult as model }\end{array}$ & $\begin{array}{l}\text { Multiple baseline across } \\
\text { participants and within } \\
\text { conversations with } \\
\text { embedded multiple } \\
\text { probes across persons, } \\
\text { settings, and stimuli }\end{array}$ & 2 & & $8-9$ & $\begin{array}{l}\text { Anecdotal: able to } \\
\text { answer questions } \\
\text { and occasionally } \\
\text { ask questions }\end{array}$ & $\checkmark$ & $\checkmark$ \\
\hline $\begin{array}{l}\text { Charlop-Christy et al. } \\
\text { (2000) }\end{array}$ & $\begin{array}{l}\text { Video modelling- } \\
\text { adult as model }\end{array}$ & $\begin{array}{l}\text { Multiple baseline } \\
\text { across participants }\end{array}$ & 1 & & 11 & $\begin{array}{l}\text { SCOSOD mental } \\
\text { age } 4 \text { years } \\
\text { and } 4 \text { months, } \\
\text { VABS: low }\end{array}$ & $\checkmark$ & $\checkmark$ \\
\hline Haymes (1995) & $\begin{array}{l}\text { Video modelling- } \\
\text { adult as model }\end{array}$ & $\begin{array}{l}\text { Multiple baseline across } \\
\text { participants and multiple } \\
\text { probes between objects }\end{array}$ & 3 & & $6-9$ & $\begin{array}{l}\text { WISC-R score: } 57-78 \text {, } \\
\text { WJ score: } 2 \text { nd } \\
\text { percentile, MLU } \\
2.21-3.39\end{array}$ & $\checkmark$ & $x$ \\
\hline $\begin{array}{l}\text { Maione and Mirenda } \\
\text { (2006) }\end{array}$ & $\begin{array}{l}\text { Video modelling- } \\
\text { adult as model }\end{array}$ & $\begin{array}{l}\text { Multiple baseline across } \\
\text { stimuli }\end{array}$ & 1 & & 5 & $\begin{array}{l}\text { CELF: age equivalence } \\
3 \text { years and } 9 \text { months }\end{array}$ & $\checkmark$ & $\checkmark$ \\
\hline Ogletree et al. (1995) & $\begin{array}{l}\text { Video modelling- } \\
\text { other as model }\end{array}$ & $\begin{array}{l}\text { Multiple baseline across } \\
\text { behaviors }\end{array}$ & & 1 & 5 & None reported & $x$ & $\checkmark$ \\
\hline $\begin{array}{l}\text { Sansosti and } \\
\text { Powell-Smith } \\
\text { (2008) }\end{array}$ & $\begin{array}{l}\text { Video modelling- } \\
\text { peer as model }\end{array}$ & $\begin{array}{l}\text { Multiple baseline across } \\
\text { participants }\end{array}$ & 3 & & $6-9$ & $\begin{array}{l}\text { WPPSI, OWLS, RIAS: } \\
\text { Oral/verbal score } \\
82-120\end{array}$ & $\checkmark$ & $\checkmark$ \\
\hline Scattone (2008) & $\begin{array}{l}\text { Video modelling- } \\
\text { adult as model }\end{array}$ & $\begin{array}{l}\text { Multiple baseline across } \\
\text { behaviors }\end{array}$ & 1 & & 9 & $\begin{array}{l}\text { K-BIT, WIAT- }- \\
\text { composite IQ } \\
\text { score } 109\end{array}$ & $\checkmark$ & $\checkmark$ \\
\hline Sherer et al. (2001) & $\begin{array}{l}\text { Video modelling- } \\
\text { self as model and } \\
\text { Video modelling } \\
\text { - peer as model }\end{array}$ & $\begin{array}{l}\text { Multiple baseline across } \\
\text { participants }\end{array}$ & 5 & & $4-11$ & $\begin{array}{l}\text { PPVT: } 2 \text { years and } 9 \\
\text { months- } 4 \text { years and } \\
2 \text { months, CARS: } \\
33.5-41.5\end{array}$ & $\checkmark$ & $\checkmark$ \\
\hline Taylor et al. (1999) & $\begin{array}{l}\text { Video modelling- } \\
\text { peer (sibling) as } \\
\text { model }\end{array}$ & $\begin{array}{l}\text { Multiple baseline } \\
\text { across stimuli }\end{array}$ & 2 & & $6-9$ & $\begin{array}{l}\text { Anecdotal: able to imitate } \\
\text { responses and make } \\
\text { comments }\end{array}$ & $\checkmark$ & $x$ \\
\hline $\begin{array}{l}\text { Tetreault and Lerman } \\
\text { (2010) }\end{array}$ & $\begin{array}{l}\text { Video modelling- } \\
\text { "point of view" }\end{array}$ & $\begin{array}{l}\text { Multiple baseline across } \\
\text { behaviors }\end{array}$ & 2 & 1 & $4-8$ & CARS: $32.5-39$ & $x$ & $\checkmark$ \\
\hline
\end{tabular}

$\checkmark$ information presented; Xinformation not presented; SIT-R Slosson Intelligence Test-Revised; VABS Vineland Adaptive Behavior Scales; PPVT Peabody Picture Vocabulary Test; WISC Weschler Intelligence Scale for Children; $K$-BIT Kaufman Brief Intelligence Test; $K$-TEA Kaufman Test of Educational Attainment; WIAT Weschler Individual Achievement Test; WJ III Woodcock Johnson III; CARS Childhood Autism Rating Scale; WPPSI Weschler Preschool and Primary Scale of Intelligence; OWLS Oral and Written Language Scales; RIAS Reynolds Intellectual Assessment Scales; SBIS Stanford Binet Intelligence Scales; Leiter Leiter International Performance Scales; CELF Clinical Evaluation of Language Fundamentals; MLU Mean Length of Utterance; SCOSOD Southern California Ordinal Scales of Development

satisfying the criteria for research of a high quality (Horner et al. 2005; Preston and Carter 2009).

\section{Treatment Integrity}

Only seven studies (28\%) (Davis et al. 2010; Maione and Mirenda 2006; Pollard et al. 2012; Sansosti and Powell-Smith
2008; Scattone 2008; Thiemann and Goldstein 2001; Tomaino 2011) provided data on treatment integrity. Where data were provided, an explanation of the data collection process and the results were presented. In most cases, a checklist was used to ensure the correct steps were followed accurately. The results ranged from 82 to $100 \%$. 
Table 2 Summary of information reported in studies using video modelling

\begin{tabular}{|c|c|c|c|c|c|c|c|c|c|}
\hline \multirow[t]{2}{*}{ Authors } & \multirow[t]{2}{*}{ Dependent variable } & \multirow[t]{2}{*}{ Setting } & \multicolumn{3}{|c|}{ Generalisation } & \multirow[t]{2}{*}{ Maintenance } & \multirow{2}{*}{$\begin{array}{l}\text { Treatment } \\
\text { integrity }\end{array}$} & \multirow{2}{*}{$\begin{array}{l}\text { Interrater } \\
\text { reliability }\end{array}$} & \multirow{2}{*}{$\begin{array}{l}\text { Presence of } \\
\text { additional } \\
\text { reinforcers }\end{array}$} \\
\hline & & & Stimuli & People & Settings & & & & \\
\hline Apple et al. (2005) & $\begin{array}{l}\text { Compliments- } \\
\text { initiating and } \\
\text { responding }\end{array}$ & Mainstream school & $x$ & $x$ & $x$ & $x$ & $x$ & $\checkmark$ & $\checkmark$ \\
\hline Buggey (2005) & Initiations & Mainstream school & $x$ & $x$ & $x$ & $\checkmark$ & $x$ & $\checkmark$ & $x$ \\
\hline $\begin{array}{l}\text { Charlop and } \\
\text { Milstein (1989) }\end{array}$ & $\begin{array}{l}\text { Appropriate } \\
\text { question asking }\end{array}$ & After school program & $\checkmark$ & $\checkmark$ & $\checkmark$ & $\checkmark$ & $x$ & $\checkmark$ & $\checkmark$ \\
\hline $\begin{array}{l}\text { Charlop-Christy } \\
\text { et al. (2000) }\end{array}$ & $\begin{array}{l}\text { Responses and } \\
\text { appropriate } \\
\text { question asking }\end{array}$ & After school program & $x$ & $x$ & $\checkmark$ & $x$ & $x$ & $\checkmark$ & $x$ \\
\hline Charlop et al. (2008) & Responses & After school program & $\checkmark$ & $\checkmark$ & $\checkmark$ & $x$ & $x$ & $\checkmark$ & $x$ \\
\hline Haymes (1995) & $\begin{array}{l}\text { Initiations, } \\
\text { responses, and } \\
\text { elaborations }\end{array}$ & After school program & $x$ & $\checkmark$ & $\checkmark$ & $x$ & $x$ & $\checkmark$ & $x$ \\
\hline $\begin{array}{l}\text { Maione and } \\
\text { Mirenda (2006) }\end{array}$ & $\begin{array}{l}\text { Initiations and } \\
\text { responses }\end{array}$ & Home & $x$ & $x$ & $x$ & $\checkmark$ & $\checkmark$ & $\checkmark$ & $x$ \\
\hline Ogletree et al. (1995) & Topic maintenance & Clinic & $x$ & $x$ & $x$ & $x$ & $x$ & $x$ & $x$ \\
\hline $\begin{array}{l}\text { Sansosti and Powell- } \\
\text { Smith (2008) }\end{array}$ & $\begin{array}{l}\text { Joining in and topic } \\
\text { maintenance }\end{array}$ & $\begin{array}{l}\text { Mainstream School- } \\
\text { Special Unit }\end{array}$ & $x$ & $x$ & $\checkmark$ & $\checkmark$ & $\checkmark$ & $\checkmark$ & $x$ \\
\hline Scattone (2008) & Initiations & Clinic & $x$ & $\checkmark$ & $\checkmark$ & $x$ & $x$ & $\checkmark$ & $x$ \\
\hline Sherer et al. (2001) & Responses & Home & $\checkmark$ & $\checkmark$ & $\checkmark$ & $\checkmark$ & $x$ & $\checkmark$ & $x$ \\
\hline Taylor et al. (1999) & Comments & Home & $x$ & $x$ & $x$ & $x$ & $x$ & $\checkmark$ & $\checkmark$ \\
\hline $\begin{array}{l}\text { Tetreault and } \\
\text { Lerman (2010) }\end{array}$ & Vocal behavior & Clinic & $\checkmark$ & $x$ & $x$ & $\checkmark$ & $x$ & $\checkmark$ & $\checkmark$ \\
\hline
\end{tabular}

\section{Social Validity}

Social validity data were provided for seven studies (28 \%); (Charlop and Milstein 1989; Davis et al. 2010; Haymes 1995; Kyparissos 1997; Lee 2007; Thiemann and Goldstein 2001; Tomaino 2011). Tomaino (2011) Lee (2007), Thiemann and Goldstein (2001), and Charlop and Milstein (1989) used a Likert scale questionnaire returning results in the middle of the range indicating a moderate rate of satisfaction in the intervention. Haymes (1995) also used a Likert scale as a measure in her study and the results returned a perception of mild improvement overall. Kyparissos (1997) asked eight teachers of children with ASD who were naïve to the study and six graduate psychology students who had no knowledge of ASD to evaluate videos of the participants pre- and post-treatment. The independent raters were asked to determine which scenes showed the participant being more engaged in conversation. Then the independent raters were asked to rate if the participants had "somewhat improved", "improved", or "greatly improved" after treatment. Eighty-seven percent of the independent raters indicated that the participants had "greatly improved" and $13 \%$ rated the participants had "improved". The process used to assess social validity was described in one study but quantitative results were not reported.

\section{Settings}

Thirteen studies were conducted in a school (seven in an inclusive school and six in a segregated setting). Eight studies were conducted at a clinic or an afterschool centre, and three were conducted at the participant's home. One study was conducted both at home and at a clinic.

\section{Dependent Variables}

A variety of individual components of conversation were measured. Non-instrumental verbal initiations were measured in 7 studies; verbal exchanges, interactions, or statements in 6 studies; responses (compliments, questions, or comments) in 11 studies; topic maintenance in 2 studies; and the number of verbal comments in 3 studies. Tetreault and Lerman (2010) investigated the use of video modelling to first make eye contact, offer a toy, and subsequently ask for the toy back. These variables were deemed to constitute a conversation as it included an initiation, an acceptance of a response from the target, and a request to engage in a play activity. More than one variable was measured in several studies.

\section{Maintenance and Generalisation}

Maintenance data was reported for 13 (52\%) studies. The range of time from the end of intervention to data collection 
Table 3 Summary of design and participants in studies using scripts

\begin{tabular}{|c|c|c|c|c|c|c|c|c|c|}
\hline \multirow[t]{2}{*}{ Authors } & \multirow[t]{2}{*}{ Script type } & \multirow[t]{2}{*}{ Fading } & \multirow[t]{2}{*}{ Design } & \multicolumn{2}{|c|}{ Participant } & \multirow[t]{2}{*}{$\begin{array}{l}\text { Age } \\
\text { (years) }\end{array}$} & \multirow[t]{2}{*}{ Participant details } & \multicolumn{2}{|c|}{$\begin{array}{l}\text { Diagnostic } \\
\text { information }\end{array}$} \\
\hline & & & & Male & Female & & & Tool & Agent \\
\hline Brown et al. (2008) & Visual & $\checkmark$ & $\begin{array}{l}\text { Multiple baseline } \\
\text { across settings }\end{array}$ & 3 & & $7-13$ & $\begin{array}{l}\text { PPVT: } 1 \text { year and } 8 \\
\text { months }-4 \text { years } \\
\text { and } 10 \text { months, } \\
\text { VABS: } 2 \text { years and } \\
11 \text { months }-5 \text { years } \\
\text { and } 5 \text { months }\end{array}$ & $\checkmark$ & $\checkmark$ \\
\hline $\begin{array}{l}\text { Charlop-Christy and } \\
\text { Kelso (2003) }\end{array}$ & Visual & $x$ & $\begin{array}{l}\text { Multiple baseline } \\
\text { across participants } \\
\text { with embedded } \\
\text { multiple probes } \\
\text { within child across } \\
\text { conversations. }\end{array}$ & 3 & & $6-7$ & $\begin{array}{l}\text { VABS: } 4 \text { years and } \\
4 \text { months, WISC-R } \\
\text { (verbal): } 60-79\end{array}$ & $\checkmark$ & $\checkmark$ \\
\hline Davis et al. (2010) & Visual & $x$ & $\begin{array}{l}\text { Multiple probe across } \\
\text { participants }\end{array}$ & 3 & & $16-17$ & $\begin{array}{l}\text { RIAS - 101, } \\
\text { CARS: } 30.5-33\end{array}$ & $\checkmark$ & $\checkmark$ \\
\hline $\begin{array}{l}\text { Krantz and } \\
\text { McClannahan } \\
\text { (1998) }\end{array}$ & Visual & $\checkmark$ & $\begin{array}{l}\text { Multiple baseline across } \\
\text { participants }\end{array}$ & 3 & & $4-5$ & SBIS IQ: $36-62$ & $\checkmark$ & \\
\hline Kyparissos (1997) & Visual & $\checkmark$ & $\begin{array}{l}\text { Multiple baseline across } \\
\text { stimuli }\end{array}$ & 2 & 1 & $15-20$ & $\begin{array}{l}\text { WJ: } 2 \text { nd }-4 \text { th } \\
\text { grade reading level }\end{array}$ & $x$ & $\checkmark$ \\
\hline Lee (2007) & Visual & $\checkmark$ & $\mathrm{ABAC}$ & 2 & 1 & $6-11$ & $\begin{array}{l}\text { Anecdotal: vocal } \\
\text { imitation and } \\
\text { answering questions }\end{array}$ & $x$ & $\checkmark$ \\
\hline Pollard et al. (2012) & Visual & $\checkmark$ & $\begin{array}{l}\text { Multiple baseline across } \\
\text { participants }\end{array}$ & 2 & 1 & $4-7$ & $\begin{array}{l}\text { Anecdotal: complex } \\
\text { language repertoire, } \\
\text { 5-7 word utterances }\end{array}$ & $x$ & $x$ \\
\hline Sarokoff et al. (2001) & Visual & $\checkmark$ & $\begin{array}{l}\text { Multiple baseline across } \\
\text { stimuli }\end{array}$ & 2 & & $8-9$ & $\begin{array}{l}\text { SBIT: borderline } \\
\text { intellectual functioning, } \\
\text { WISC: moderate } \\
\text { range of mental } \\
\text { retardation }\end{array}$ & $x$ & $x$ \\
\hline Stevenson et al. (2000) & Audio & $\checkmark$ & $\begin{array}{l}\text { Multiple baseline across } \\
\text { activities plus } \\
\text { multiple probes } \\
\text { across participants }\end{array}$ & 4 & & $10-15$ & $\begin{array}{l}\text { PPVT: } 2 \text { years and } \\
7 \text { months- } 6 \text { years } \\
\text { and } 9 \text { months }\end{array}$ & $\checkmark$ & $\checkmark$ \\
\hline $\begin{array}{l}\text { Thiemann and } \\
\text { Goldstein (2001) }\end{array}$ & $\begin{array}{l}\text { Visual plus video } \\
\text { feedback }\end{array}$ & $x$ & $\begin{array}{l}\text { Multiple baseline across } \\
\text { participants }\end{array}$ & 4 & & $6-12$ & $\begin{array}{l}\text { CARS: mild to } \\
\text { moderate-severe } \\
\text { range }\end{array}$ & $x$ & $x$ \\
\hline Tomaino (2011) & Visual & $x$ & $\begin{array}{l}\text { Multiple baseline across } \\
\text { participants }\end{array}$ & 3 & 3 & $5-10$ & $\begin{array}{l}\text { PPVT: } 4 \text { years and } \\
3 \text { months- } 10 \text { years } \\
\text { and } 1 \text { month, } \\
\text { EVT: } 5-11 \text { years } \\
5 \text { months, } \\
\text { VABS: } 3 \text { years and } \\
5 \text { months }-6 \text { years } \\
\text { and } 6 \text { months }\end{array}$ & $x$ & $x$ \\
\hline Wichnick et al. (2010) & Audio & $\checkmark$ & $\begin{array}{l}\text { Multiple baseline across } \\
\text { participants }\end{array}$ & 2 & 1 & $5-7$ & $\begin{array}{l}\text { Anecdotal: limited } \\
\text { conversational and } \\
\text { spontaneous language }\end{array}$ & $x$ & $\checkmark$ \\
\hline
\end{tabular}

$\checkmark$ information presented; Xinformation not presented; SIT-R Slosson Intelligence Test Revised; VABS Vineland Adaptive Behavior Scales; PPVT Peabody Picture Vocabulary Test; WISC Weschler Intelligence Scale for Children; $K$-BIT Kaufman Brief Intelligence Test; $K$-TEA Kaufman Test of Educational Attainment; WIAT Weschler Individual Achievement Test; WJ III Woodcock Johnson III; CARS Childhood Autism Rating Scale; WPPSI Weschler Preschool and Primary Scale of Intelligence; OWLS Oral and Written Language Scales; RIAS Reynolds Intellectual Assessment Scales; SBIS Stanford Binet Intelligence Scales; Leiter Leiter International Performance Scales; CELF Clinical Evaluation of Language Fundamentals

for maintenance ranged from 1 day (Sansosti and PowellSmith 2008) to 15 months (Charlop and Milstein 1989). Generalisation data across stimulus, persons, and settings were only reported in seven $(28 \%)$ studies. There were no generalisation data reported in seven $(28 \%)$ studies. Generalisation data for only one domain were reported in four 
Table 4 Summary of information reported in studies using scripts

\begin{tabular}{|c|c|c|c|c|c|c|c|c|c|}
\hline \multirow[t]{2}{*}{ Authors } & \multirow[t]{2}{*}{ Dependent variable } & \multirow[t]{2}{*}{ Setting } & \multicolumn{3}{|c|}{ Generalisation } & \multirow[t]{2}{*}{ Maintenance } & \multirow{2}{*}{$\begin{array}{l}\text { Treatment } \\
\text { integrity }\end{array}$} & \multirow{2}{*}{$\begin{array}{l}\text { Interrater } \\
\text { reliability }\end{array}$} & \multirow{2}{*}{$\begin{array}{l}\text { Presence } \\
\text { of additional } \\
\text { reinforcers }\end{array}$} \\
\hline & & & Stimuli & People & Settings & & & & \\
\hline Brown et al. (2008) & Interactions & $\begin{array}{l}\text { Mainstream } \\
\text { school }\end{array}$ & $\checkmark$ & $x$ & $\checkmark$ & $x$ & $x$ & $\checkmark$ & $\checkmark$ \\
\hline $\begin{array}{l}\text { Charlop-Christy and } \\
\text { Kelso (2003) }\end{array}$ & Responses & $\begin{array}{l}\text { After school } \\
\text { program }\end{array}$ & $\checkmark$ & $\checkmark$ & $\checkmark$ & $x$ & $x$ & $\checkmark$ & $x$ \\
\hline Davis et al. (2010) & $\begin{array}{l}\text { Other focused } \\
\text { conversation-eye } \\
\text { contact, listening } \\
\text { to partner, questions } \\
\text { and comments }\end{array}$ & $\begin{array}{l}\text { Mainstream } \\
\text { school }\end{array}$ & $x$ & $\checkmark$ & $\checkmark$ & $x$ & $\checkmark$ & $\checkmark$ & $x$ \\
\hline $\begin{array}{l}\text { Krantz and } \\
\text { McClannahan } \\
\text { (1998) }\end{array}$ & $\begin{array}{l}\text { Interactions and } \\
\text { elaborations }\end{array}$ & Special school & $\checkmark$ & $\checkmark$ & $x$ & $\checkmark$ & $x$ & $\checkmark$ & $x$ \\
\hline Kyparissos (1997) & Verbal exchanges & Special school & $\checkmark$ & $x$ & $x$ & $x$ & $\checkmark$ & $\checkmark$ & $\checkmark$ \\
\hline Lee (2007) & Responses & Special school & $\checkmark$ & $\checkmark$ & $\checkmark$ & $x$ & $x$ & $\checkmark$ & $\checkmark$ \\
\hline Pollard et al. (2012) & Initiations & $\begin{array}{l}\text { Mainstream } \\
\text { school }\end{array}$ & $\checkmark$ & $\checkmark$ & $\checkmark$ & $\checkmark$ & $\checkmark$ & $\checkmark$ & $x$ \\
\hline $\begin{array}{l}\text { Sarokoff, Taylor, and } \\
\text { Poulson (2001) }\end{array}$ & Statements & Special school & $\checkmark$ & $\checkmark$ & $x$ & $\checkmark$ & $x$ & $\checkmark$ & $x$ \\
\hline Stevenson et al. (2000) & Interactions & Special school & $x$ & $x$ & $x$ & $\checkmark$ & $x$ & $\checkmark$ & $x$ \\
\hline $\begin{array}{l}\text { Thiemann and } \\
\text { Goldstein (2001) }\end{array}$ & Initiations & $\begin{array}{l}\text { Mainstream } \\
\text { school }\end{array}$ & $\checkmark$ & $x$ & $\checkmark$ & $\checkmark$ & $\checkmark$ & $\checkmark$ & $x$ \\
\hline Tomaino (2011) & Responses & $\begin{array}{l}\text { Clinic and } \\
\text { home }\end{array}$ & $\checkmark$ & $\checkmark$ & $\checkmark$ & $\checkmark$ & $\checkmark$ & $\checkmark$ & $x$ \\
\hline Wichnick et al. (2010) & Responses & Special school & $x$ & $x$ & $x$ & $\checkmark$ & $x$ & $\checkmark$ & $\checkmark$ \\
\hline
\end{tabular}

(16\%) studies, and data for two of the three possible domains were reported in the remaining seven studies ( $28 \%)$.

\section{Design and Demonstration of Experimental Control}

A multiple baseline design was employed in 23 (92\%) studies. A multiple baseline across participants design was used in 14 studies, a multiple baseline across behaviors or stimuli design was used in 8 studies and a multiple baseline across settings design was employed in 1 study. A combination of multiple baseline across participants design with alternating treatments design was employed in one study, a multiple probe technique was embedded within a multiple baseline design in two studies, and a multiple probe design was used in two studies. The researchers in one study implemented an ABAC design.

Studies using a multiple baseline, $\mathrm{ABAB}$, or alternating treatments with counterbalancing were considered to allow for a minimum of three demonstrations of experimental control at different points in time. However, studies using $\mathrm{AB}, \mathrm{ABA}$, and changing criterion designs were not considered to allow requisite demonstrations of control (Horner et al. 2005). Of the 25 studies, 23 were considered to potentially allow demonstration of adequate control. Of these, 15 studies were judged to provide the minimum three demonstrations of control. Thus, only $60 \%$ of the studies reviewed used designs that were considered as technically capable of providing the requisite three demonstrations of experimental control at different points in time and provided a clear demonstration of control.

Charlop and Milstein (1989) presented a complex multiple probe design where treatment effects were interpreted as demonstrating control, and improvements prior to the introduction of treatment were interpreted as generalisation. A fundamental premise underlying the rationale for the demonstration of experimental control in multiple baseline and probe designs is that baselines are independent and that intervention in one baseline should not affect others. Thus, the reported generalisation in this study may more appropriately be considered as a failure to demonstrate experimental control.

\section{Presence of Additional Reinforcers}

Additional supports in the form of verbal praise or tokens that could be exchanged for edibles or access to preferred toys were provided in eight studies. The effect of this supplementary intervention, however, could only be isolated in one study (Apple et al. 2005).

\section{PND and PEM}

PND and PEM statistics were calculated for all graphed data. For ease of reporting and comparison, the mean PND and PEM data for the interventions are presented in Tables 5 and 6. 
Video Modelling Video-modelled interventions had an overall PND of 72 including reversal data (range 41.2-100) and 72.2 without reversal data (range 41.2-100). The treatment-only statistic is marginally different at 71.4 (range $31.2-100$ ) and 71.7 (range 33-100), respectively, indicating that the inclusion of generalisation data and maintenance data was substantially consistent with treatment-only data. According to Scruggs and Mastropieri (1998) this indicates that, as a whole, a videomodelled intervention used to teach conversation was on the borderline between a questionable and effective outcome.

The PEM statistic for video-modelled studies was 83 with a range of 59.9 to 100 . The treatment-only statistic was 82.1 (range 42.2-100). This statistic is considerably higher than the PND and may be due to the presence of outlying baseline data points. Six interventions (Charlop and Milstein 1989; Charlop-Christy et al. 2000; Maione and Mirenda 2006; Ogletree et al. 1995; Scattone 2008; Taylor et al. 1999) were shown to be highly effective, scoring above 90 . It is important to note that the article by Charlop and Milstein (1989) only included graphed data for baseline, generalisation, and maintenance. There was a brief intervention phase, but no intervention data were presented. This absence of data may have inflated the metrics calculated for this study.

Based on the PEM metric, none of the studies reviewed fell below the acceptable threshold. There were only small differences between the effectiveness of using a peer, an adult, or the participant as a model; however, the use of a point-of-view video and a cartoon as a model were ineffective in the studies in which they were employed.

A comparison between the studies with additional supports embedded within the treatment and those without indicated that there was a relatively limited difference overall. Interventions with embedded reinforcement had a mean PND of 66.8 and PEM of 77.7 compared with a PND of 73.5 and PEM of 84.3 for interventions with no artificial reinforcement. The study by Apple et al. (2005) graphed the artificial reinforcement phase separately so a calculation was done for the intervention phase without reinforcement and a separate calculation for the initial intervention plus the phase with the additional reinforcement. As might be expected, the metrics for the treatment with additional reinforcement was higher (PND and PEM=68.3) than that without additional reinforcement (PND and PEM=50), indicating that the additional reinforcement contributed to the measured effects.

Scripts The overall PND was 75.8 (range 41.5-100) and the PEM was 84 (range 71.6-100), which were consistent with an effective treatment (Scruggs and Mastropieri 1998). Three studies scored higher than 90, putting them in the highly effective range, with one study (Davis et al. 2010) scoring 100 on both PND and PEM. A complete summary of data (including data with reversals) is presented in Table 5. The difference between the PND with reversals and the PND without reversals was only 0.3 . Similarly, the difference between the PEM including reversals and excluding reversals was only 0.5 . While this is a minimal overall difference the results should be interpreted cautiously as only one study (Lee 2007) used a reversal design and the inclusion of reversals decreased the overall study PND by 3.7 and the PEM by 6.3 .

There were two types of scripts used in the studies reviewed: audio-presented scripts and visual scripts (either written or pictorial). The mean PND and PEM for audio scripts was 93.7 and 93.9, respectively. The treatment-only statistic was 85.5 for both PND and PEM. The higher values for pooled data indicates that audio scripts contributed to the generalisation and maintenance of the variables measured. In comparison, visual scripts showed more moderate study aggregate PND and PEM results at 72.2 and 82.1, respectively. The result for treatment-only data was 75.4 (PND) and 83.3 (PEM). A further comparison was done between scripts that were faded and scripts that were not faded. Interventions that faded the use of scripts systematically had a study aggregate mean PND of 77 and PEM of 81.5, compared to scripts that were not faded at 73.4 (PND) and 89.1 (PEM).

\section{Discussion}

The purpose of this review was to examine the efficacy of video modelling and scripts in teaching conversational skills to people with ASD. The information provided on the abilities of the participants was diverse, ranging from anecdotal descriptions of verbal abilities to comprehensive formal assessments. The degree of autistic symptomatology was only reported in four studies, and only four participants were considered as "severely" autistic. Without an appropriate assessment of the degree of impairment, it is difficult to compare the relative efficacy of the treatments reviewed and this information is crucial when researchers seek to replicate the studies conducted. Single-subject designs are often criticised for limited external validity making it important to provide adequate participant descriptions. Where formal measures of cognitive functioning were reported, the majority of the participants were either within or just outside the "normal" range of intellectual functioning, which may indicate that conversational skills are considered by researchers as higher order skills that are most appropriately taught to higher functioning individuals.

Video modelling was on the borderline between questionably effective and effective for teaching conversational skills with an overall PND of 72 and a somewhat higher PEM of 83 . There were limited differences between the apparent effectiveness of different types of video models with the exception of point-of-view modelling, which did not appear to be effective in the one study in which it was employed. The use of a 
Table 5 PND and PEM for individual studies

\begin{tabular}{|c|c|c|c|c|c|c|c|}
\hline \multirow[b]{2}{*}{ Studies } & & \multicolumn{2}{|c|}{ Treatment phase only } & \multicolumn{2}{|c|}{ Full study (including maintenance and generalisation) } & \multicolumn{2}{|c|}{ Additional data } \\
\hline & & PND & PEM & PND & PEM & PND & PEM \\
\hline Apple et al. (2005) & & 68.3 & 68.3 & N/A & N/A & N/A & N/A \\
\hline Brown et al. (2008) & & 91.1 & 92.4 & 90.6 & 91.6 & $\mathrm{~N} / \mathrm{A}$ & N/A \\
\hline Buggey (2005) & & 80 & 85.7 & 86.5 & 90.4 & N/A & N/A \\
\hline Charlop and Milstein (1989) & & N/A & N/A & 73.9 & 93.7 & $\mathrm{~N} / \mathrm{A}$ & N/A \\
\hline Charlop-Christy and Kelso (2003) & & 83.3 & 95.2 & 65.4 & 88.5 & N/A & N/A \\
\hline Charlop-Christy et al. (2000) & & 100 & 100 & 100 & 100 & N/A & N/A \\
\hline Charlop et al. (2008) & & 84 & 88 & 62.2 & 78.4 & N/A & N/A \\
\hline Davis et al. (2010) & & 100 & 100 & 100 & 100 & $\mathrm{~N} / \mathrm{A}$ & N/A \\
\hline Haymes (1995) & & 33 & 60.2 & 41.2 & 61.5 & N/A & N/A \\
\hline Krantz and McClannahan (1998) & & 66.3 & 69.7 & 73 & 73 & $\mathrm{~N} / \mathrm{A}$ & N/A \\
\hline Kyparissos (1997) & & 71 & 73 & 69.4 & 71.6 & $\mathrm{~N} / \mathrm{A}$ & N/A \\
\hline Lee (2007) & & 71.7 & 87.5 & 62.7 & 82.9 & $59^{\mathrm{b}}$ & $76.5^{\mathrm{b}}$ \\
\hline Maione and Mirenda (2006) & & 54.9 & 74.5 & 73.2 & 92.2 & $66.7^{\mathrm{a}}$ & $89.8^{\mathrm{a}}$ \\
\hline Ogletree et al. (1995) & & 62.5 & 100 & N/A & N/A & N/A & N/A \\
\hline Pollard et al. (2012) & & 50 & 54.2 & 67.7 & 75.3 & N/A & N/A \\
\hline Sansosti and Powell-Smith (2008) & & 73.1 & 88.5 & 71.8 & 88.5 & $80^{\mathrm{a}}$ & $92.5^{\mathrm{a}}$ \\
\hline Sarokoff et al. (2001) & & 69.1 & 75.9 & N/A & N/A & N/A & N/A \\
\hline Scattone (2008) & & 90 & 100 & 90.9 & 100 & N/A & $\mathrm{N} / \mathrm{A}$ \\
\hline \multirow[t]{2}{*}{ Sherer et al. (2001) } & Self as model & 69.9 & 69.9 & 62.1 & 62.1 & $69.9^{\mathrm{a}}$ & $69.9^{\mathrm{a}}$ \\
\hline & Other as model & 73.8 & 73.8 & 64.5 & 64.5 & 73.8 & 73.8 \\
\hline Stevenson et al. (2000) & & 81 & 81 & 97.5 & 97.9 & N/A & N/A \\
\hline Taylor et al. (1999) & & 87.4 & 90.74 & N/A & N/A & N/A & N/A \\
\hline Tetreault and Lerman (2010) & & 36.2 & 48.9 & 51.1 & 64.2 & $47.6^{\mathrm{b}}$ & $59.9^{\mathrm{b}}$ \\
\hline Thiemann and Goldstein (2001) & & 48.72 & 89.7 & 41.5 & 81.5 & N/A & $\mathrm{N} / \mathrm{A}$ \\
\hline Tomaino (2011) & & 100 & 100 & 86.6 & 86.6 & N/A & N/A \\
\hline Wichnick et al. (2010) & & 89.9 & 89.9 & N/A & $\mathrm{N} / \mathrm{A}$ & N/A & N/A \\
\hline
\end{tabular}

${ }^{\text {a }}$ Treatment only data with prompts

${ }^{\mathrm{b}}$ Pooled data with reversals

point-of-view perspective may have been confusing for the participant as it is inconsistent with the cultural convention of presenting a video from a third party perspective. Nevertheless, any interpretations made based on the type of model used to facilitate behavior change should be made cautiously as the number of studies is limited.

Scripts were used as the primary intervention in 12 studies. Overall, the PND (75.8) and PEM (84) suggest that scripts

Table 6 Mean PND and PEM for scripts and video modelling interventions

\begin{tabular}{|c|c|c|c|c|c|c|c|c|c|c|}
\hline & & \multicolumn{4}{|l|}{ Scripts } & \multicolumn{5}{|l|}{ Video modelling } \\
\hline & & $\begin{array}{l}\text { Audio } \\
(n=2)\end{array}$ & $\begin{array}{l}\text { Visual, written or } \\
\text { pictorial }(n=10)\end{array}$ & $\begin{array}{l}\text { Faded } \\
(n=8)\end{array}$ & $\begin{array}{l}\text { Not faded } \\
(n=4)\end{array}$ & $\begin{array}{l}\text { Peer as model } \\
\left(n=4^{\mathrm{a}}\right)\end{array}$ & $\begin{array}{l}\text { Adult as } \\
\text { model }(n=6)\end{array}$ & $\begin{array}{l}\text { Self as model } \\
\left(n=2^{\mathrm{a}}\right)\end{array}$ & $\begin{array}{l}\text { Point of view } \\
(n=1)\end{array}$ & $\begin{array}{l}\text { Other } \\
(n=1)\end{array}$ \\
\hline \multirow[t]{2}{*}{ Pooled data } & PND & 93.7 & 72.2 & 77.0 & 73.4 & 68.2 & 77 & 74.3 & 47.6 & N/A \\
\hline & PEM & 93.9 & 82.1 & 81.5 & 89.1 & 73.8 & 89 & 76.2 & 59.9 & N/A \\
\hline \multirow[t]{2}{*}{ Treatment data only } & PND & 85.5 & 75.4 & 73.4 & 83 & 71.7 & 76.5 & 75.0 & 31.2 & 63.5 \\
\hline & PEM & 85.5 & 83.3 & 77.3 & 96.3 & 76.9 & 87.8 & 77.8 & 42.2 & 100 \\
\hline
\end{tabular}

${ }^{a}$ Total number of studies using video modelling=13, one study compared a video of the participant (self) with a video of a peer 
may be an effective intervention for conversational skills. Audio-recorded scripts were implemented in 2 studies, and written scripts were used in 10 studies. Thiemann and Goldstein (2001) incorporated video feedback with a textand visual-based script intervention. A comparison of the PND and PEM of the audio scripts and written scripts suggested that audio scripts (PND 93.7, PEM 93.9) may be more effective than written scripts (PND 71, PEM 82.3). It is possible that audio scripts may be more easily imitated vocally by the participants and may better simulate the cues a partner may give in a more natural environment. A comparison of the treatment-only portion (excluding generalisation and maintenance) indicated some difference between audio scripts (PND 85.5, PEM 85.5) and video-modelling interventions (PND 71.4, PEM 82.1). While there is a disparity for PND, the similar PEM values do not suggest a meaningful difference in the relative effectiveness of audio scripts compared to video modelled interventions. However, only two studies used audio scripts and therefore, these findings should be interpreted with extreme caution.

The PND and PEM statistics conflicted when a comparison was made between scripts that were faded and those that were not. The PND showed that faded interventions (77) were similar in effectiveness to interventions that were not faded (73.4), but the PEM statistic suggested the possibility of marginally better performance with the non-faded scripts (89) compared to faded scripts (81.5). Both PND and PEM statistics indicated that scripts that were faded allowed for marginally better generalisation of skills as the treatmentonly result was lower than the result that included generalisation and maintenance phases. The gradual fading of the intervention may have contributed to the maintenance and generalisation of the skills learnt during treatment. However, as only four studies (Charlop-Christy and Kelso 2003; Davis et al. 2010; Pollard et al. 2012; Thiemann and Goldstein 2001) systematically faded the scripts used in the treatment phase, it would be prudent to limit the conclusions drawn from such a limited number of studies, and further research in this area would be warranted.

Treatment integrity data were only reported in seven studies. Where information was available, the data showed that treatments were conducted appropriately, but the absence of this data in the majority of the studies makes it difficult to determine if the interventions were administered as designed. Other authors of reviews on interventions in the autism field have found a similar paucity of data in this regard, which indicates that treatment integrity should be carefully monitored (Bellini and Akullian 2007; Preston and Carter 2009; Reynhout and Carter 2006).

Generalisation or maintenance data were reported for 18 studies. The results were mixed with some studies reporting generalisation of skills and several reporting minimal or no generalisation (Brown et al. 2008; Charlop and Milstein 1989;
Lee 2007; Sherer et al. 2001; Taylor et al. 1999; Tetreault and Lerman 2010). However, several generalisation probes were executed in very similar environments to the original interventions or using similar stimuli. For example, Sansosti and Powell-Smith (2008) conducted generalisation probes in a fenced-in playground behind the participant's school and the intervention probes were conducted in a fenced-in playground within the school grounds. Similarly, Charlop et al. (2009) used a black and red yo-yo instead of a blue, red, and green yo-yo to measure stimulus generalisation. In only one study (Brown et al. 2008) were participants taken to different community locations to collect generalisation data. In summary, although generalisation was often examined, the nature of the generalisation was often minor and, consequently, the observed degree of generalisation limited. Similarly, where maintenance data was taken, it was often completed soon after the treatment phase had ended. Only one study undertook ongoing long-term maintenance probes at 1-, 2-, 3-, 6-, and 15-months post intervention (Charlop and Milstein 1989).

Conversation involves a multitude of skills and there was a considerable variation in the dependent variables examined. The skills examined ranged from basic skills such as initiating or responding to more complex variables such as maintaining a topic or joining in a conversation. The more complex conversational skills such as topic maintenance and appropriate exchanges, however, were only addressed in four studies. The operational definitions also varied between studies, although the variables measured were similar. For example, Wichnick et al. (2010) considered a response to be an unprompted word if uttered within $5 \mathrm{~s}$ of an initiation by a peer, whereas Charlop and Milstein (1989); Charlop-Christy et al. (2000), Charlop et al. (2009), and Sherer et al. (2001) defined a response as a correct answer and a return question. These differences complicate the task of drawing conclusions from these data. For example, although video modelling interventions had a lower mean PND and PEM than interventions utilising scripts, the dependent variables measured were more diverse and complex, as topic maintenance was examined in two studies (Ogletree et al. 1995; Sansosti and PowellSmith 2008).

Both scripted and unscripted verbal exchanges were measured in several of the studies reviewed. Scripted verbal exchanges were reported to have increased in all of the studies, but the number of unscripted or novel interactions was not reported as increasing to the same degree. Where gains in unscripted interactions were reported, the definition of an "unscripted" interaction was often only either a change in the referent object or verbatim repetitions of a script used in a previous session. It is important to note that in several studies, once scripts were faded, all verbalisations were recorded as unscripted thereby inflating the gains reported. Any inferences drawn from these gains in unscripted verbalisations would have to be cautiously interpreted. 
Artificial reinforcers were implemented in several studies (Apple et al. 2005; Brown et al. 2008; Charlop and Milstein 1989; Kyparissos 1997; Lee 2007; Tetreault and Lerman 2010; Wichnick et al. 2010) to motivate the participants. Tokens, points, or food that are delivered contingently upon correct responses can serve to confound interpretation of the results as it is difficult to disentangle the effects of the intervention from those of the artificial reinforcement. In one study (Apple et al. 2005), once the food reinforcer was withdrawn, the level of initiations returned to baseline levels showing that peer interactions were insufficient as a natural reinforcement to maintain the behavior.

\section{Limitations}

A number of limitations of this review need to be acknowledged. The studies included in this review were limited to the verbal content of conversation and did not address the other, equally important, non-verbal aspects such as facial expression, intonation, and expressive gestures. There are also limited data available on the degree of impairment of the participants making it impossible to examine relative efficacy of different interventions for participants with similar degrees of impairments. The relatively small corpus of research also limited the ability to examine moderator variables.

\section{Future Research}

Although it has been shown that children with ASD tend to initiate and respond to adults more often than to other children (Hauck et al. 1995; Jackson et al. 2003; McHale et al. 1980) the findings of this review indicate that interventions using peer partners may be more effective. In future research, it may be appropriate to compare the relative efficacy of employing similar aged peers versus adults as conversational partners within the same intervention and dependent variables in order to determine if this leads to generalisation of any skills learnt to other peers. Children with ASD rarely talk about things "outside the immediate situation" (Capps et al. 1998; Rutter 1978), hence, it may also be advisable to conduct research on interventions seeking to improve conversational skills on topics not immediately present. As children with ASD have been found to "repeat prior comments and questions verbatim" and do not extend topics by offering new and relevant information (Capps et al. 1998), it would also be beneficial to conduct further research into whether interventions would be successful in improving these aspects of conversation.

The authors in most of the studies examined relatively superficial aspects of conversation such as initiating or responding appropriately to a conversation partner. Effective conversations are comprised of more than initiating and responding, therefore further research could examine whether video modelling or scripts can improve the ability of children with ASD to engage in more complex conversational skills such as topic maintenance, topic shifts, or monitoring of a partner's level of interest. In addition, future research on video modelling could pay further attention to the non-verbal dimensions of conversation as individuals rely on more than the verbal utterances from a partner to glean the meanings in social communication. As the research available focuses on participants who are higher functioning, it would be appropriate to examine the extent to which the interventions could be applied to individuals who are lower functioning. More generally, researchers need to provide more detailed participant descriptions, including measures of the degree of autistic symptomatology.

\section{Conclusion}

Based on the research reviewed, it would appear that video modelling was in the marginally effective range and scripts were in the low effective range in improving the conversational skills of children and adolescents with ASD, when applied to basic skills such as initiating and responding. Although data were limited, there were suggestions that within scripted interventions, the use of audio scripts were more effective than visual scripts. Further, scripts that were faded provided better generalisation results. The simplicity of a scripted intervention is appealing, but additional research is required to address the more complex conversational skills.

\section{References}

American Psychiatric Association. (2000). Diagnostic and statistical manual of mental disorders : DSM-IV-TR (4th ed.). Washington, DC: American Psychiatric Association.

Apple, A. L., Billingsley, F., Schwartz, I. S., \& Carr, E. G. (2005). Effects of video modeling alone and with self-management on complimentgiving behaviors of children with high-functioning ASD. Journal of Positive Behavior Interventions, 7, 33-46. doi:10.1177/ 10983007050070010401.

Baron-Cohen, S. (1995). Mindblindness: an essay on autism and theory of mind. Cambridge, MA: MIT Press.

Bellini, S., \& Akullian, J. (2007). A meta-analysis of video modeling and video self-modeling interventions for children and adolescents with autism spectrum disorders. Exceptional Children, 73(3), 264-287.

Brown, J. L., Krantz, P. J., McClannahan, L. E., \& Poulson, C. L. (2008). Using script fading to promote natural environment stimulus control of verbal interactions among youths with autism. Research in Autism Spectrum Disorders, 2, 480-497. doi:10.1016/j.rasd.2007.08.006.

Buggey, T. (2005). Video self-modeling applications with students with autism spectrum disorder in a small private school setting. Focus on Autism and Other Developmental Disabilities, 20, 52-63. doi:10. 1177/10883576050200010501.

Capps, L., Kehres, J., \& Sigman, M. (1998). Conversational abilities among children with autism and children with developmental delays. Autism, 2, 325-344. doi:10.1177/1362361398024002. 
Carpenter, M., \& Tomasello, M. (2000). Joint attention, cultural learning, and language acquisition: implications for children with autism. In A. M. Wetherby \& B. M. Prizant (Eds.), Autism spectrum disorders: A transactional developmental perspective (pp. 31-54). Baltimore: Paul H Brookes.

Carter, M. (2013). Reconsidering overlap-based measures for quantitative synthesis of single-subject data: what they tell us and what they don't. Behavior Modification. doi:10.1177/0145445513476609.

Carter, A. S., Davis, N. O., Klin, A., \& Volkmar, F. R. (2005). Social development in autism. In F. R. Volkmar, R. Paul, A. Klin, \& D. J. Cohen (Eds.), Handbook of autism and pervasive developmental disorders (Vol. 1, pp. 312-334). Hoboken: Wiley.

Charlop, M. H., \& Milstein, J. P. (1989). Teaching autistic children conversational speech using video modeling. Journal of Applied Behavior Analysis, 22, 275-285. doi:10.1901/jaba.1989.22-275.

Charlop, M. H., Gilmore, L., \& Chang, G. T. (2009). Using video modeling to increase variation in the conversation of children with autism. Journal of Special Education Technology, 23, 47-66.

Charlop, M. H., Dennis, B., Carpenter, M. H., \& Greenberg, A. L. (2010). Teaching socially expressive behaviors to children with autism through video modeling. Education \& Treatment of Children, 33, 371-393.

Charlop-Christy, M. H., \& Kelso, S. E. (2003). Teaching children with autism conversational speech using a cue card/written script program. Education \& Treatment of Children, 26, 108-127.

Charlop-Christy, M. H., Le, L., \& Freeman, K. A. (2000). A comparison of video modeling with in vivo modeling for teaching children with autism. Journal of Autism and Developmental Disorders, 30, 537552.

Church, C., Alisanski, S., \& Amanullah, S. (2000). The social, behavioral, and academic experiences of children with Asperger syndrome. Focus on Autism \& Other Developmental Disabilities, 15, 12. doi: $10.1177 / 108835760001500102$.

D'Ateno, P., Mangiapanello, K., \& Taylor, B. A. (2003). Using video modeling to teach complex play sequences to a preschooler with autism. Journal of Positive Behavior Interventions, 5(1), 5-11.

Davis, K. M., Boon, R. T., Cihak, D. F., \& Fore, C., III. (2010). Power cards to improve conversational skills in adolescents with Asperger syndrome. Focus on Autism and Other Developmental Disabilities, 25, 12-22. doi:10.1177/1088357609354299.

Dixon, D. R., Tarbox, J., \& Najdowski, A. (2009). Social skills in autism spectrum disorders. In J. L. Matson (Ed.), Social behavior and skills in children (pp. 117-140). New York: Springer Science + Business Media.

Ferraioli, S. J., \& Harris, S. L. (2011). Treatments to increase social awareness and social skills Evidence-based practices and treatments for children with autism (pp. 171-196). New York: Springer Science + Business Media.

Fine, J., Bartolucci, G., Szatmari, P., \& Ginsberg, G. (1994). Cohesive discourse in pervasive developmental disorders. Journal of Autism \& Developmental Disorders, 24, 315-329.

Gage, N. A., \& Lewis, T. J. (2012). Analysis of effect for single-case design research. Journal of Applied Sport Psychology, 25(1), 46-60. doi:10.1080/10413200.2012.660673.

Hauck, M., Fein, D., Waterhouse, L., \& Feinstein, C. (1995). Social initiations by autistic children to adults and other children. Journal of Autism and Developmental Disorders, 25, 579-595. doi:10.1007/ BF02178189.

Haymes, L. K. (1995). Using video modeling to improve conversation skills with autistic children: a comparison of obsessions and nonobsessions as topics. Doctoral dissertation, The Claremont Graduate School, Claremont, CA. Retrieved from http://ovidsp.ovid.com/ ovidweb.cgi? $\mathrm{T}=\mathrm{JS} \& \mathrm{CSC}=\mathrm{Y} \& \mathrm{NEWS}=\mathrm{N} \& \mathrm{PAGE}=$ fulltext $\& \mathrm{D}=$ psyc3\&AN=1995-95011-189 (12-B)

Horner, R. H., Carr, E. G., Halle, J., McGee, G., Odom, S., \& Wolery, M. (2005). The use of single-subject research to identify evidence- based practice in special education. Exceptional Children, 71, $165-179$.

Jackson, C. T., Fein, D., Wolf, J., Jones, G., Hauck, M., Waterhouse, L., \& Feinstein, C. (2003). Responses and sustained interactions in children with mental retardation and autism. Journal of Autism and Developmental Disorders, 33, 115-121.

Klinger, L. G., \& Williams, A. (2009). Cognitive-behavioral interventions for students with autism spectrum disorders. In M. J. Mayer, J. E. Lochman, \& R. Van Acker (Eds.), Cognitive-behavioral interventions for emotional and behavioral disorders: school-based practice (pp. 328-362). New York: Guilford Press.

Krantz, P., \& McClannahan, L. (1998). Social interaction skills for children with autism: a script-fading procedure for beginning readers. Journal of Applied Behavior Analysis, 31, 191-202. doi: 10.1901/jaba.1998.31-191.

Kyparissos, N. (1997). Extending conversations among adolescent peers with autism. Doctoral dissertation, The City University of New York, New York. Retrieved from http://ovidsp.ovid.com/ovidweb. cgi? $\mathrm{T}=\mathrm{JS} \& \mathrm{CSC}=\mathrm{Y} \& \mathrm{NEWS}=\mathrm{N} \& \mathrm{PAGE}=$ fulltext $\& \mathrm{D}=\mathrm{psyc} 3 \& \mathrm{AN}=$ 1997-95008-091 (10-B)

Lee, R. (2007). The effects of script-fading and a Lag-1 schedule on varied social responding in children with autism. Doctoral dissertation, The City University of New York, New York. Retrieved from http://ovidsp.ovid.com/ovidweb.cgi? $\mathrm{T}=\mathrm{JS} \& \mathrm{CSC}=\mathrm{Y} \& \mathrm{NEWS}=$ $\mathrm{N} \& \mathrm{PAGE}=$ fulltext $\& \mathrm{D}=$ psyc5\&AN=2007-99004-067 (8-B)

Ma, H.-H. (2006). An alternative method for quantitative synthesis of single-subject researches: percentage of data points exceeding the median. Behavior Modification, 30, 598-617. doi:10.1177/ 0145445504272974

MacDonald, R., Clark, M., Garrigan, E., \& Vangala, M. (2005). Using video modeling to teach pretend play to children with autism. Behavioral Interventions, 20, 225-238.

Machalicek, W., Davis, T., O’Reilly, M., Beretvas, N., Sigafoos, J., Lancioni, G., \& Edrisinha, C. (2008). Teaching social skills in school settings. In J. K. Luiselli, D. C. Russo, W. P. Christian, \& S. M. Wilczynski (Eds.), Effective practices for children with autism (pp. 269-298). New York: Oxford University Press.

Maione, L., \& Mirenda, P. (2006). Effects of video modeling and video feedback on peer-directed social language skills of a child with autism. Journal of Positive Behavior Interventions, 8, 106-118. doi:10.1177/10983007060080020201.

Matson, J. L., Matson, M. L., \& Rivet, T. T. (2007). Social-skills treatments for children with autism spectrum disorders: an overview. Behavior Modification, 31, 682-707. doi:10.1177/ 0145445507301650.

McHale, S. M., Simeonsson, R. J., Marcus, L. M., \& Olley, J. (1980). The social and symbolic quality of autistic children's communication. Journal of Autism and Developmental Disorders, 10, 299-310. doi: 10.1007/BF02408289.

Ogletree, B. T., Fischer, M. A., \& Sprouse, J. (1995). An innovative language treatment for a child with high-functioning autism. Focus on Autistic Behavior, 10(3), 1-10. doi:10.1177/ 108835769501000301.

Parker, R. I., \& Hagan-Burke, S. (2007). Median-based overlap analysis for single case data: a second study. Behavior Modification, 31, 919-936. doi:10.1177/0145445507303452.

Pollard, J. S., Betz, A. M., \& Higbee, T. S. (2012). Script fading to promote unscripted bids for joint attention in children with autism. Journal of Applied Behavior Analysis, 45(2), 387-393.

Preston, D., \& Carter, M. (2009). A review of the efficacy of the picture exchange communication system intervention. Journal of Autism and Developmental Disorders, 39, 1471-1486. doi:10.1007/ s10803-009-0763-y.

Reynhout, G., \& Carter, M. (2006). Social storiestm for children with disabilities. Journal of Autism and Developmental Disorders, 36, 445-469. doi:10.1007/s10803-006-0086-1. 
Rubin, E., \& Lennon, L. (2004). Challenges in social communication in asperger syndrome and high-functioning autism. Topics in Language Disorders, 24, 271-285. doi:10.1097/00011363200410000-00005.

Rutter, M. (1978). Diagnosis and definition of childhood autism. Journal of Autism and Developmental Disorders, 8, 139-161. doi:10.1007/ bf01537863.

Sansosti, F. J., \& Powell-Smith, K. A. (2008). Using computer-presented social stories and video models to increase the social communication skills of children with high-functioning autism spectrum disorders. Journal of Positive Behavior Interventions, 10, 162-178. doi:10. 1177/1098300708316259.

Sarokoff, R. A., Taylor, B. A., \& Poulson, C. L. (2001). Teaching children with autism to engage in conversational exchanges: script fading with embedded textual stimuli. Journal of Applied Behavior Analysis, 34, 81-84. doi:10.1901/jaba.2001.34-81.

Scattone, D. (2007). Social skills interventions for children with autism. Psychology in the Schools, 44, 717-726. doi:10.1002/pits.20260.

Scattone, D. (2008). Enhancing the conversation skills of a boy with Asperger's disorder through social stories TM and video modeling. Journal of Autism and Developmental Disorders, 38, 395-400. doi: 10.1007/s10803-007-0392-2.

Scruggs, T. E., \& Mastropieri, M. A. (1998). Summarizing single-subject research: issues and applications. Behavior Modification, 22, 221242. doi:10.1177/01454455980223001.

Scruggs, T. E., Mastropieri, M. A., \& Casto, G. (1987). The quantitative synthesis of single-subject research: methodology and validation. RASE: Remedial \& Special Education, 8(2), 24-33.

Shea, V., \& Mesibov, G. B. (2005). Adolescents and adults with autism. In F. R. Volkmar, R. Paul, A. Klin, \& D. J. Cohen (Eds.), Handbook of autism and pervasive developmental disorders: Diagnosis, development, neurobiology, and behavior (Vol. 1, pp. 288-311). Hoboken: Wiley.

Sherer, M., Pierce, K. L., Paredes, S., Kisacky, K. L., Ingersoll, B., \& Schreibman, L. (2001). Enhancing conversation skills in children with autism via video technology: which is better, "self" or "other" as a model? Behavior Modification, 25, 140-158. doi:10.1177/ 0145445501251008.

Stevenson, C. L., Krantz, P. J., \& McClannahan, L. E. (2000). Social interaction skills for children with autism: a script-fading procedure for nonreaders. Behavioral Interventions, 15, 1-20. doi:10.1002/ (SICI)1099-078X(200001/03)15:1<1::AID-BIN41>3.0.CO;2-V.

Stichter, J. P., Randolph, J., Gage, N., \& Schmidt, C. (2007). A review of recommended social competency programs for students with autism spectrum disorders. Exceptionality, 15, 219-232. doi:10.1080/ 09362830701655758.
Taylor, B. A., Levin, L., \& Jasper, S. (1999). Increasing play-related statements in children with autism toward their siblings: effects of video modeling. Journal of Developmental and Physical Disabilities, 11, 253-264.

Tetreault, A. S., \& Lerman, D. C. (2010). Teaching social skills to children with autism using point-of-view video modeling. Education \& Treatment of Children, 33, 395-419. doi:10.1353/ etc. 0.0105 .

Thiemann, K. S., \& Goldstein, H. (2001). Social stories, written text cues, and video feedback: effects on social communication of children with autism. Journal of Applied Behavior Analysis, 34, 425-446. doi:10.1901/jaba.2001.34-425.

Tomaino, M. A. E. (2011). Teaching conversation to children with autism: Assessment of the efficacy of a parent-implemented script procedure. 3449924 Ph.D., The Claremont Graduate University, Ann Arbor. Retrieved from http://simsrad.net.ocs.mq.edu.au/login? http://search.proquest.com/docview/863813780?accountid= 12219http://HH9MG3RW5Q.search.serialssolutions.com/?ctx ver=Z39.88-2004\&ctx_enc $=$ info:ofi/enc:UTF-8\&rfr_id $=$ info:sid/ ProQuest+Dissertations $+\% 26+$ Theses + Full+Text\&rft_val_fmt $=$ info:ofi/fmt:kev:mtx: dissertation\&rft.genre $=$ dissertations $+\%$ $26+$ theses $\&$ rft.jtitle $=\&$ rft.atitle $=\& \mathrm{rft} . \mathrm{au}=$ Tomaino $\%$ $2 \mathrm{C}+$ Melaura + Andree+Erickson\&rft.aulast $=$ Tomaino\&rft.aufirst $=$ Melaura + Andree \&rft.date $=2011-01-01 \&$ rft. volume $=\&$ rft.issue $=$ $\&$ rft.spage $=\&$ rft.isbn $=9781124577074 \&$ rft.btitle $=\&$ rft.title $=$ Teaching + conversation+to+children+with+autism $\%$ $3 \mathrm{~A}+$ Assessment + of + the ProQuest Dissertations \& Theses Full Text database

Twachtman-Cullen, D. (2000). More able children with autism spectrum disorders: sociocommunicative challenges and guidelines for enhancing abilities. In A. M. Wetherby \& B. M. Prizant (Eds.), Autism spectrum disorders: A transactional developmental perspective (pp. 225-249). Baltimore: Paul H. Brooks.

Weiss, M. J., LaRue, R. H., \& Newcomer, A. (2009). Social skills and autism: Understanding and addressing the deficits. In J. L. Matson (Ed.), Applied behavior analysis for children with autism spectrum disorders (pp. 129-144). New York: Springer Science + Business Media.

Wichnick, A. M., Vener, S. M., Pyrtek, M., \& Poulson, C. L. (2010). The effect of a script-fading procedure on responses to peer initiations among young children with autism. Research in Autism Spectrum Disorders, 4, 290-299. doi:10.1016/j.rasd.2009.09.016.

Williams White, S., Keonig, K., \& Scahill, L. (2007). Social skills development in children with autism spectrum disorders: a review of the intervention research. Journal of Autism and Developmental Disorders, 37, 1858-1868. 